\title{
Design and Analysis of a Novel Wall-Climbing Robot Mechanism
}

\section{Weiguang Dong ${ }^{1,2, a}$, Hongguang Wang ${ }^{1, b}$, Aihua Liu ${ }^{1}$ and Zhenhui Li ${ }^{1,2}$}

${ }^{1}$ State Key Laboratory of Robotics, Shenyang Institute of Automation Chinese Academy of Sciences, Shenyang 110016, China

${ }^{2}$ Graduate School of the Chinese Academy of Sciences, Beijing 100039, China

adongweiguang@sia.cn, bhgwang@sia.cn

\section{Keywords: wall-climbing mechanism; hybrid locomotion; kinematics}

\begin{abstract}
A novel wall-climbing robot mechanism designed for anti-hijacking task is presented. This mechanism consists of a negative pressure adhesion module, a vacuum suction module and a planetary-gear train. The design of biped-wheel hybrid locomotion mechanism, with the advantages of wheeled robots and legged robots, allows the robot to move fast and cross over obstacles easily. This design qualifies the robot for the motion of moving straight, turning in plane and crossing between inclined surfaces. Then the kinematics equations are derived and the locomotion modes are analyzed. Many experiments have been implemented and the results prove that the robot has such characteristics as rapid speed, excellent transition ability between inclined surfaces and curved surface adaptability. Therefore, this novel wall-climbing mechanism could be used for the application of inspection, surveillance and reconnaissance.
\end{abstract}

\section{Introduction}

In recent years, wall-climbing robots have received increasing attention for their applications in dangerous environments like exterior of high-rise buildings, airplanes, or nuclear facilities. Climbing robots can increase operation efficiency, protect human health and save cost. For example, in military field, they can implement tasks such as aircraft inspection, surveillance and reconnaissance and target acquisition; in chemical field, they can be used for maintenance and sand blasting of storage tanks, inspection of pipelines or gas ducts and spray painting. Therefore, the research of wall-climbing robots has significant value $[1,2]$.

It is well-known that an excellent mechanism plays an important role for the robot performance. Recently, the mechanism receives more and more attention by research and development institutions $[3,4]$. Basically, the research on the wall-climbing robot has focused on two aspects: locomotion mechanism and adhesion mechanism. From the locomotion point of view, wall-climbing robots can be divided into four categories: legged structure, tracked structure, wheeled structure and combined structure $[1,5]$. The robot employing legged locomotion mechanism usually adapts vacuum suction technique or magnetic adhesion technique, including robots by Paul [6] and the robot Shigeo [7]. These robots have good trafficability and adsorption capacity with low working noise. However, their moving speed is usually slow. The robot equipped with tracked structure usually adapts magnetic adhesion technique or vacuum suction technique, including robots by Shen [8] and Kim [9]. These robots have good adsorption capacity and fast moving speed. However, their ability of surmounting obstacles is relatively low. The robot using wheeled locomotion mechanism usually employs negative pressure adhesion technique, including robots by Xiao [10] and the robot Alicia [11]. These robots are easy to control and have relatively fast moving speed. However, they have low trafficability and consume a lot of energy with high working noise. The final type is the robot combining the aforementioned locomotive mechanisms together to improve the climbing ability, including robot by $\mathrm{Fu}$ [12]. This kind of robot appears currently and indicates a new trend of the development of climbing robot.

Generally, the anti-hijacking task presents higher request on moving speed, trafficability and curved surface adaptability. According to the research fruits of formers, a novel wall-climbing robot mechanism has been developed. This mechanism consists of a negative pressure adhesion module, a 
vacuum suction module and a planetary-gear train. It adapts wheel-leg hybrid locomotion mechanism, with the advantages of wheeled robots and legged robots, allows the robot to move fast and cross over obstacles easily. The kinematics analysis and experiments show that this wall-climbing mechanism has such characteristics as rapid speed, excellent transition ability between inclined surfaces and curved surface adaptability and satisfies the basic requirements of anti-terrorist reconnaissance missions.

\section{Mechanism Configuration of the Robot}

The basic design concept of the robot is to form a novel wall-climbing mechanism by combining two different locomotive mechanisms and two different adhesion manners. This wall-climbing mechanism consists of a negative pressure adhesion module, a vacuum suction module and a specially designed planetary-gear train. The idea of this design is shown in Fig.1.The assembly model of the mechanism is shown in Fig.2. The negative pressure module is composed of a centrifugal fan, a big suction cup, a 3-wheel mechanism, and a big gear as a crust. The vacuum suction module is composed of a linear motion unit, a vacuum suction device, a supporting wheel and a big gear as a crust.

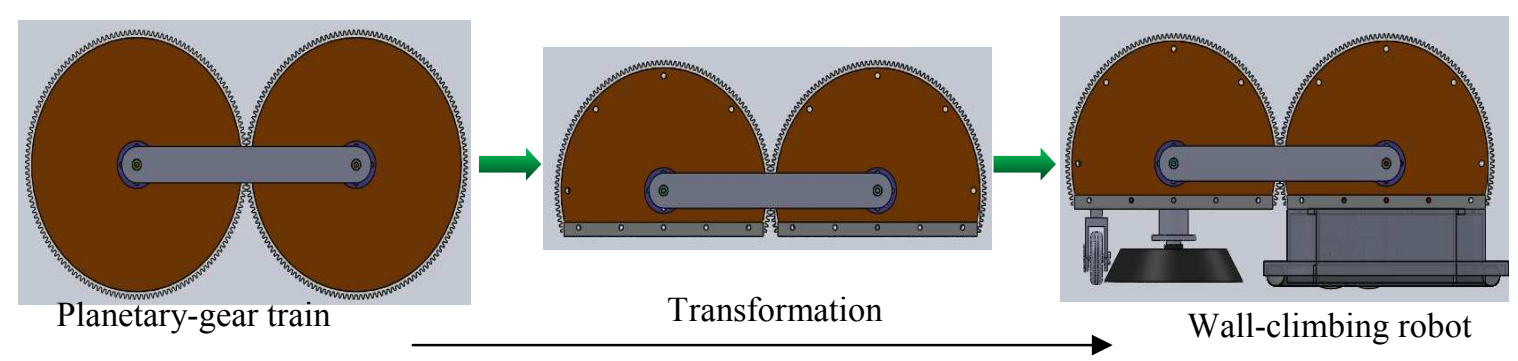

Fig. 1. The idea of design of planetary-gear train

This climbing mechanism has four degrees of freedom that can be seen from the sketch of the mechanism, as shown in Fig.3. The linear motion unit has one degree of freedom and is driven by a DC motor to realize the motion of stretching out and drawing back of the vacuum sucker. The wheeled locomotion mechanism is composed of two driving wheels and a castor wheel and has two degrees of freedom. By adjusting the speed of the two driving wheels, the robot can realized the motion of moving straight and turning in plane. The planetary-gear train has one degree of freedom, which means the traversing motion of the robot can be realized when driven by only one DC motor. The movement of the planetary-gear train in combination with the movement of the sucker can realized the traversing between inclined surfaces. Besides, this robot adopts two kinds of adhesion techniques, negative pressure adhesion technique and vacuum suction adhesion technique, to work in combination with climbing mechanism.

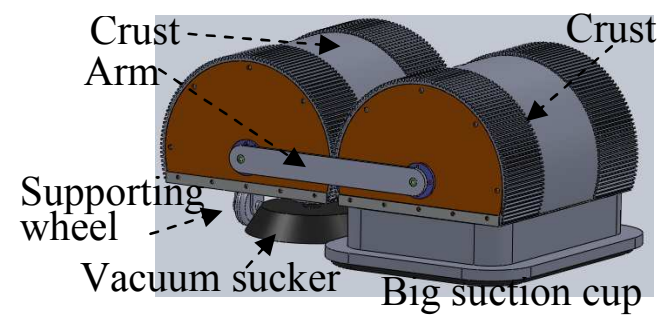

Fig. 2. The assembled view of the robot

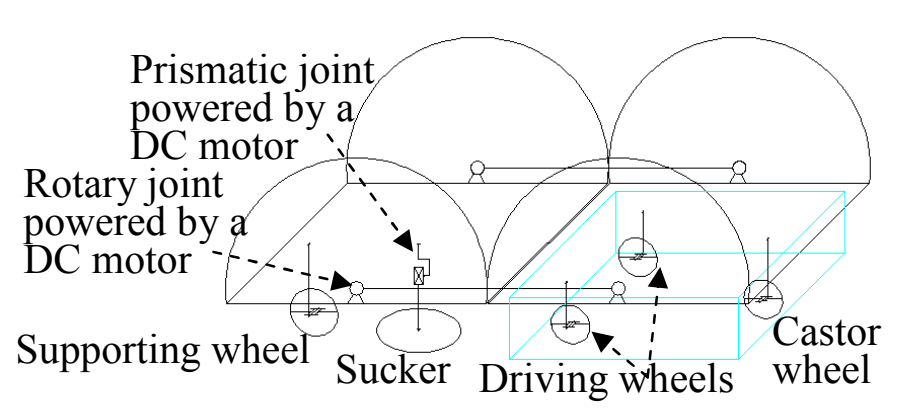

Fig. 3. The kinematic sketch of the robot mechanism 


\section{Kinematics Analysis}

D-H Notation. According to the design, this wall-climbing mechanism has three major locomotion modes of moving straight, turning and traversing between inclined surfaces. The kinematic analysis provided in the following subsections focus on the traversing mode using Denavit-Hartenberg Notation to describe mechanisms of the robot. Assignment of coordinate systems is dependent upon which module is sticking to surface. In this paper, the coordinate frames are assigned when the negative pressure adhesion module is sticking to surface. Assignment of the coordinate systems is illustrated in Fig.4. The parameters to the links are shown in Table 1.
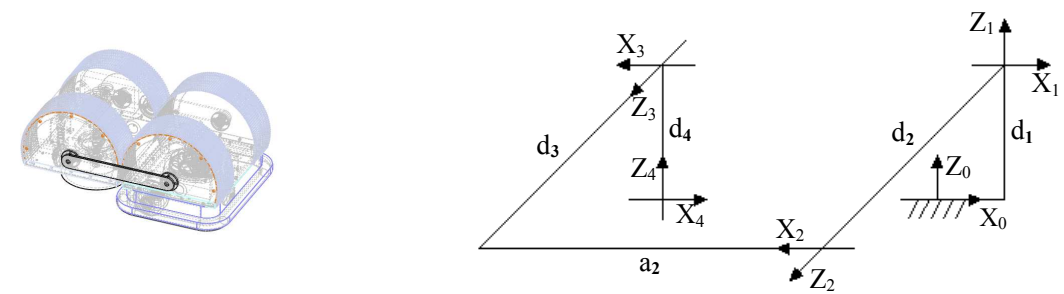

Fig. 4. The sketch of the mechanism and the coordinates frames Table 1 The link paramenters of the mechanism

\begin{tabular}{cccccc}
\hline joint & $\mathrm{a}_{\mathrm{i}-1}[\mathrm{~mm}]$ & $\alpha_{\mathrm{i}-1}\left[^{\circ}\right]$ & $\mathrm{d}_{\mathrm{i}}[\mathrm{mm}]$ & $\theta_{\mathrm{i}}\left[^{\circ}\right]$ & Range of joint \\
\hline 1 & $\mathrm{a}_{0}$ & 0 & $\mathrm{~d}_{1}$ & 0 & 0 \\
2 & 0 & $90^{\circ}$ & $\mathrm{d}_{2}$ & $\theta_{2}$ & $-20^{\circ}-200^{\circ}$ \\
3 & $\mathrm{a}_{2}$ & 0 & $-\mathrm{d}_{3}$ & $\theta_{3}$ & $-20^{\circ}-200^{\circ}$ \\
4 & 0 & $-90^{\circ}$ & $-\mathrm{d}_{4}$ & 0 & $\pm 10 \mathrm{~mm}$ \\
\hline
\end{tabular}

We start by defining the reference frame, frame $\{0\}$, the origin of which is in the middle of the ground contact points of the two driving wheels. The z-axis is parallel with the axle of driving wheel. The $\mathrm{x}$-axis points to the moving direction of the robot. The frame $\{4\}$, the final frame, is attached to the vacuum sucker, and the origin of frame $\{4\}$ is in the center of the vacuum sucker. $\mathrm{a}_{\mathrm{i}}\{\mathrm{i}=1,2,3,4\}$ represents the length of each link. $\theta_{2}, \theta_{3}, \mathrm{~d}_{4}$ are joint variables, and $a_{2}$ represents length of the rod. The sun gear and the planet pinion are designed to have same diameter, so there is equation $\theta_{2}=\theta_{3}$ based on the transmission principle of planetary-gear train.

Kinematics Equation. The relationship between the vacuum sucker and negative pressure adhesion module can be expressed by ${ }_{4}^{0} T$, as in Eq. 1, including position and orientation of the vacuum sucker to the reference frame.

$$
\begin{aligned}
& { }_{1}^{0} T=\left[\begin{array}{cccc}
1 & 0 & 0 & a_{0} \\
0 & 1 & 0 & 0 \\
0 & 0 & 1 & d_{1} \\
0 & 0 & 0 & 1
\end{array}\right],{ }_{2}^{1} T=\left[\begin{array}{cccc}
c \theta_{2} & -s \theta_{2} & 0 & 0 \\
0 & 0 & -1 & -d_{2} \\
s \theta_{2} & c \theta_{2} & 0 & 0 \\
0 & 0 & 0 & 1
\end{array}\right],{ }_{3}^{2} T=\left[\begin{array}{cccc}
c \theta_{3} & -s \theta_{3} & 0 & a_{2} \\
s \theta_{3} & c \theta_{3} & 0 & 0 \\
0 & 0 & 1 & -d_{2} \\
0 & 0 & 0 & 1
\end{array}\right],{ }_{4}^{3} T=\left[\begin{array}{cccc}
1 & 0 & 0 & 0 \\
0 & 1 & 0 & 0 \\
0 & 0 & 1 & -d_{4} \\
0 & 0 & 0 & 1
\end{array}\right], \\
& { }_{4}^{0} T={ }_{1}^{0} T_{2}^{1} T_{3}^{2} T_{4}^{3} T=\left[\begin{array}{cccc}
n_{x} & s_{x} & a_{x} & p_{x} \\
n_{y} & s_{y} & a_{y} & p_{y} \\
n_{z} & s_{z} & a_{z} & p_{z} \\
0 & 0 & 0 & 1
\end{array}\right]=\left[\begin{array}{cccc}
\left(c \theta_{2}\right)^{2}-\left(s \theta_{2}\right)^{2} & -2 s \theta_{2} c \theta_{2} & 0 & -d_{4} 2 s \theta_{2} c \theta_{2}+a_{2} c \theta_{2}+a_{0} \\
0 & 0 & -1 & 0 \\
2 s \theta_{2} c \theta_{2} & \left(c \theta_{2}\right)^{2}-\left(s \theta_{2}\right)^{2} & 0 & \left(c \theta_{2}\right)^{2}-\left(s \theta_{2}\right)^{2}+a_{2} s \theta_{2}+d_{1} \\
0 & 0 & 0 & 1
\end{array}\right],
\end{aligned}
$$

where, $c \theta=\cos \theta, s \theta=\sin \theta$.

Inverse Kinematics Equation. The inverse kinematics equation is derived from the Eq.1. According to transformation matrix ${ }_{4}^{0} T$, the joint variables can be calculated. If $n_{z}>0$, the plus-or-minus sign of $\cos \theta_{2}$ and $\sin \theta_{2}$ is identical, so $\theta_{2} \in\left(0,90^{\circ}\right) \cup\left(180^{\circ}, 200^{\circ}\right)$; If $n_{z}<0$, the plus-or-minus sign of $\cos \theta_{2}$ and $\sin \theta_{2}$ is opposite, $\operatorname{so} \theta_{2} \in\left(-20^{\circ}, 0\right) \cup\left(90^{\circ}, 180^{\circ}\right)$. 


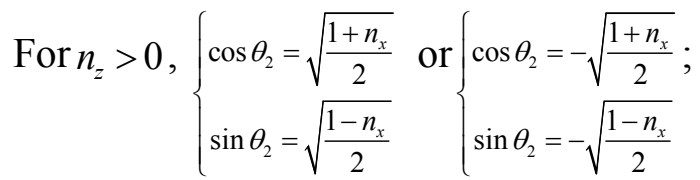

$$
\begin{aligned}
& \text { For } n_{z}<0,\left\{\begin{array} { l } 
{ \operatorname { c o s } \theta _ { 2 } = \sqrt { \frac { 1 + n _ { x } } { 2 } } \text { or } } \\
{ \operatorname { s i n } \theta _ { 2 } = - \sqrt { \frac { 1 - n _ { x } } { 2 } } }
\end{array} \left\{\begin{array}{l}
\cos \theta_{2}=-\sqrt{\frac{1+n_{x}}{2}} \\
\sin \theta_{2}=\sqrt{\frac{1-n_{x}}{2}}
\end{array}\right.\right. \\
& d_{4}=\frac{a_{2} c \theta_{2}+a_{0}-p_{x}}{2 s \theta_{2} c \theta_{2}} \text {. }
\end{aligned}
$$

\section{Locomotion Modes Analysis}

Viewed from the design of hybrid locomotion mechanism, wheeled locomotion is the primary pattern of movement that plays a major role in fast moving. The traversing mode is mainly used to step over obstacles and cross between inclined surfaces. The hybrid locomotion mechanism possesses advantages of the two locomotion mechanism and improves the robot's adaptability to environment.

Wheeled Locomotion. The main function of wheeled locomotion is rectilinear motion and rotation. Besides, by changing the speed of driving wheels of the 3-wheel locomotion mechanism, the robot can realize the motion of spinning and turning easily. When the speed of the two driving wheels keeps equal and in the same direction, the robot will realize rectilinear motion. When the speed of the two driving wheels keeps same but in the opposite direction, the robot will spin around, and the rotating center is located in the middle of the ground contact points of the two driving wheels, as shown in Fig.5.1. By changing the speed of the two driving wheels, there are different turning radiuses, as shown in Fig.5.2. Turning radius $\mathrm{R}$ is as Eq. 5.

$$
R=\frac{L\left(v_{1}+v_{2}\right)}{2\left(v_{2}-v_{1}\right)} .
$$
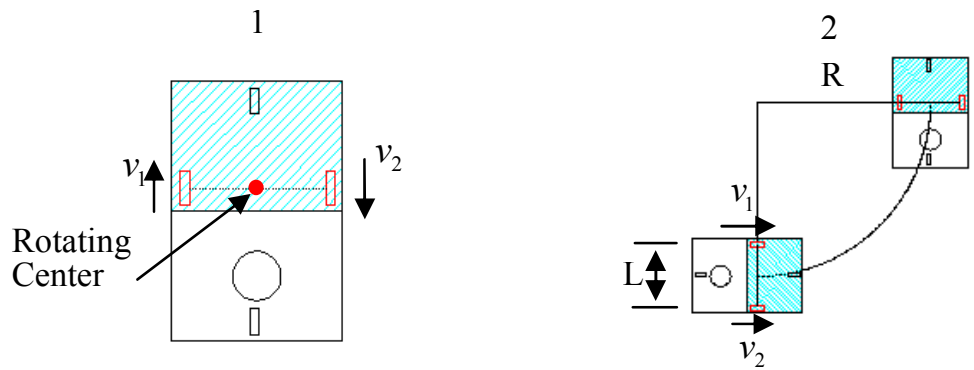

Fig. 5. Spinning and Turning

Traversing Motion. Traversing motion is mainly used to step over obstacles and cross between inclined surfaces, and its process is relatively complicated. The movement sequence provided in the following subsections includes crossing between ground and wall and stepping over obstacle on wall.

Crossing from Ground to Wall. The gait can be divided into the following order, as shown in Fig.6 (Shaded areas indicate the state of adsorbing).

a) The robot moves to an appropriate place, as shown in Fig.6.1;

b) The robot rotates the vacuum suction module and aligns vacuum sucker with the target surface, as shown in Fig.6.2;

c) The robot approachs the target surface with its vacuum sucker, when pressure is established, it closes the centrifugal pump, as shown in Fig.6.3;

d)The robot rotates the negative pressure adhesion module until the big cup is in close contact with the surface, when pressure is established, it releases its vacuum sucker, as shown in Fig.6.4;

Stepping over Obstacle on Wall. The gait can be divided into the following order, as shown in Fig.7 (Shaded areas indicate the state of adsorbing). 
a) The robot moves to an appropriate place, as shown in Fig.7.1;

b) The robot rotates the vacuum suction module and aligns vacuum sucker with the target surface, as shown in Fig.7.2;

c) The robot stretches the vacuum sucker until vacuum sucker is in close contact with the surface, when pressure is established; it closes the centrifugal pump, as shown in Fig.7.3;

d) The robot rotates the negative pressure adhesion module until the big cup is in close contact with the surface, when negative pressure is established, it releases its vacuum sucker, as shown in Fig.7.4;
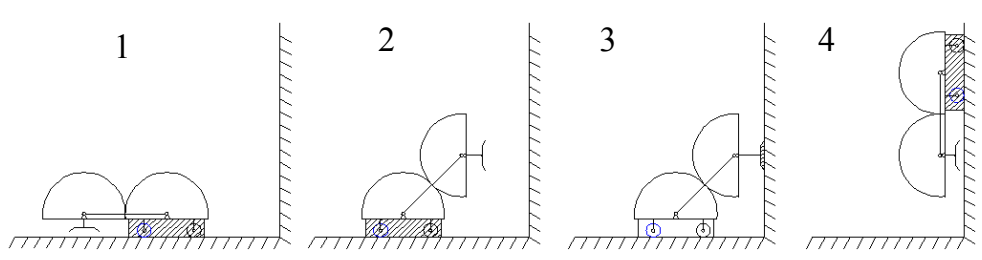

Fig. 6. Movement of crossing from ground to wall

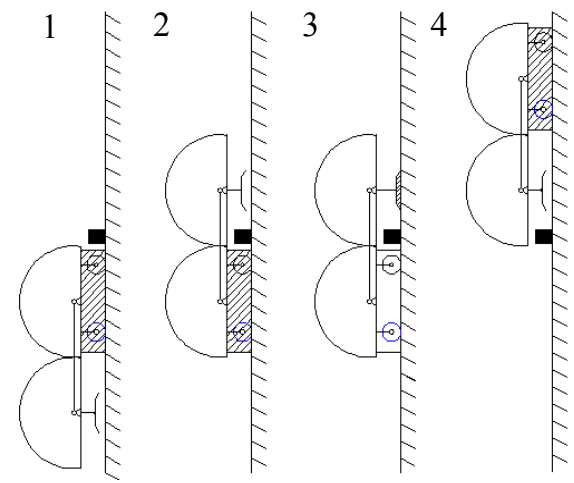

Fig. 7. Movement of stepping over obstacle

\section{Experiments}

To verify the feasibility and effectiveness of the presented wall-climbing mechanism, a prototype is designed and implemented and some experiments are carried out. The experimental content includes moving on flat surfaces of various materials and the exterior surface of an aircraft. Meanwhile, the trafficability and the ability of crossing between inclined surfaces are also verified. The specifications of the robot are listed in Table 2.

Table 2 Specifications of the robot

\begin{tabular}{ccccc}
\hline $\begin{array}{c}\text { Weight } \\
{[\mathrm{kg}]}\end{array}$ & \multicolumn{2}{c}{ Maximum speed $[\mathrm{m} / \mathrm{min}]$} & Size $\left[\mathrm{mm}^{3}\right]$ & $\begin{array}{c}\text { Endurance } \\
{[\mathrm{mins}]}\end{array}$ \\
\cline { 2 - 3 } & surface & $\begin{array}{c}\text { In vertical } \\
\text { surface }\end{array}$ & $298 * 212 * 140$ & 45 \\
\hline 1.9 & 5 & 4 & 290 . \\
\hline
\end{tabular}

Moving on Surfaces. The robot can move on many kinds of flat surfaces: wood, metal, glass. Meanwhile, the robot can stick to surface in any angle position. Fig.8.1 shows the robot can move on wooden door and its speed reaches to $4 \mathrm{~m} / \mathrm{min}$. The robot can move on curved surface even when the smallest radius of curvature is $1.5 \mathrm{~m}$. The robot can move safely on the outer surface of an aircraft, as shown in Fig. 8.2(the aircraft is B-2223 from Civil Aviation Administration of China). The radius of curvature of the aircraft is about $2.5 \mathrm{~m}$.

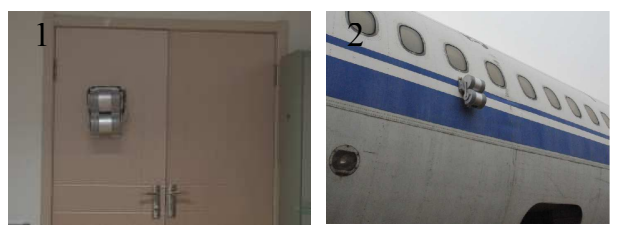

Fig. 8. Robot's movement on many kind of surfaces

Crossing from Ground to Wall. By combining two kinds of locomotive mechanisms and two types of adhesion techniques, the robot can cross between inclined surfaces easily. Experiments prove that the robot can cross from ground to a surface when the interior angle between the ground and the surface is within the range of $90^{\circ}$ to $180^{\circ}$ and the exterior angle is within the range of $0^{\circ}$ to $20^{\circ}$. A sequence of photos illustrating the robot crossing from ground to vertical wall can be seen in Fig.9. The average time needed for this process is less than 25 seconds. 


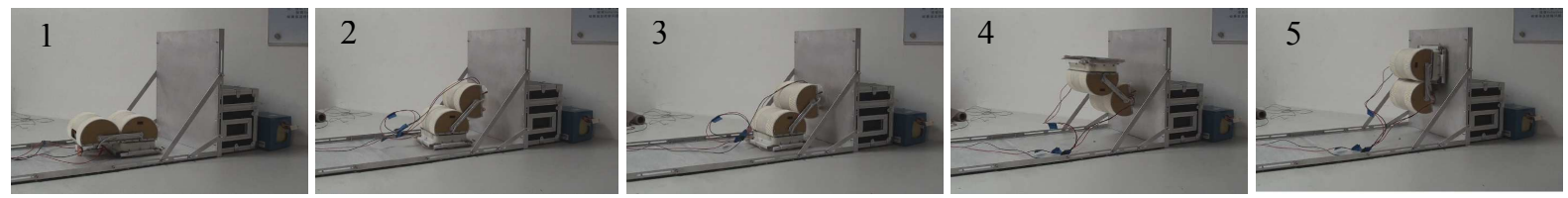

Fig. 9. Robot's movement of crossing from ground to wall

\section{Conclusion}

The design and analysis of a novel wall-climbing mechanism for anti-hijacking task have been presented. The wheel-leg hybrid locomotion mechanism, with the advantages of wheeled robots and legged robots, allows the robot to move fast and cross over obstacles easily. A specially designed planetary-gear train combines the two modules of the mechanism, which improves the motion performances of the robot. Besides, this robot adopts two kinds of adhesion techniques-negative pressure adhesion technique and vacuum suction adhesion technique, which work in combination with climbing mechanism. The results of experiment prove that the robot has such characteristics as rapid speed, excellent transition ability between inclined surfaces and curved surface adaptability. In the future research, improved centrifugal pump will be incorporated to enhance the adhesion performance with lower noise. The sensor system will also be improved to permit the robot to sense the environments accurately.

\section{Acknowledgment}

This research is supported in part by the National High-Tech Research and Development (863) Plan (No. 2009AA04Z2171). Special thanks to Xiaodong Chen, Chunhong Xing and Fengren Ging who have been instrumental to the very timely completion of this robot.

\section{References}

[1] B. Chu, K. Jung, C. Han and D. Hong, A Survey of Climbing Robots: Locomotion and Adhesion, Journal of Precision Engineering and Manufacturing, 11(2010), 633-647.

[2] B. Shores and M. Minor, Design, Kinematic Analysis, and Quasi-Steady Control of a Morphic Rolling Disk Biped Climbing Robot, presented at IEEE Int. Conf. on Robotics and Automation, Barcelona, Spain, 2005.

[3] R. L. Tummala, R. Mukherjee, N. Xi, and et al, Climbing the walls, IEEE Robotics and Automation Magazine, 9(2002) 10-19.

[4] W. Brockmann and F. Mosch, CLIMBING AND WALKING ROBOTS, presented at Int. Conf. on Climbing and Walking Robots- CLAWAR2004, Madrid, Spain, 2004.

[5] S. P. Krosuri and M. A. Minor, A multifunctional hybrid hip joint for improved adaptability in miniature climbing robots, presented at IEEE Int. Conf. on Robotics and Automation, Taipei, Taiwan, 2003.

[6] P.G. Backes, Y. Bar-Cohen, and B. Joffe, The multifunction automated crawling system (MACS), presented at IEEE Int. Conf. on Robotics and Automation, Albuquerque, USA, 1997.

[7] S. Hirose, A. Nagakubo, and R. Toyama, Machine That Can Walk and Climb on Floors, Walls and Ceilings, presented at Fifth International Conference on Advanced Robotics, Pisa, Italy,1991.

[8] W. Shen, J. Gu and Y. Shen, Permanent Magnetic System Design for the Wall-climbing Robot, presented at IEEE Int. Conf. on Mechatronics \& Automation, Niagara Falls, Canada, 2005.

[9] H. Kim, D. Kim, H.Yang, and et al, Development of a wall-climbing robot using a tracked wheel mechanism, Journal of Mechanical Science and Technology, 22 (2008) 1490 1498.

[10] Xiao, M. Minor, H. Dulimarta, N. Xi, R. Mukherjee, and R. Tummala, Modeling and control of under-actuated miniature crawler robot, presented at IEEE/RSJ Int. Conf. on Intelligent Robots and Systems, Maui, USA, 2001.

[11] D. Longo and G. Muscato, The Alicia3 Climbing Robot: A Three-Module Robot for Automatic Wall Inspection, IEEE Robotics and Automation Magazine, 13(2006) 42-50.

[12] Yili. Fu, Zhihai. Li, and Shuguo. Wang, A Wheel-leg Hybrid Wall Climbing Robot with Multi-surface Locomotion Ability, presented at IEEE Int. Conf. on Mechatronics and Automation, Kagawa, Japan, 2008. 